ELSEVIER

\title{
Clinical Differentiation of Respiratory Nursing Diagnoses among Children with Acute Respiratory Infection
}

\author{
Livia Maia Pascoal RN, PhDa , \\ Marcos Venícios de Oliveira Lopes RN, PhD (Statistician) ${ }^{\mathbf{b}, *}$, \\ Viviane Martins da Silva RN, PhD ${ }^{b}$, Daniel Bruno Resende Chaves RN, MsD ${ }^{b}$, \\ Beatriz Amorim Beltrão RN, MsD ${ }^{b}$, Marília Mendes Nunes $\mathrm{RN}^{\mathrm{b}}$, \\ Karine Kerla Maia de Moura RN ${ }^{\text {b }}$
}

\author{
${ }^{\mathrm{a}}$ Federal University of Maranhão in Imperatriz, Brazil \\ ${ }^{\mathrm{b}}$ Federal University of Ceará in Fortaleza, Brazil
}

Received 14 May 2015; revised 27 June 2015; accepted 6 August 2015

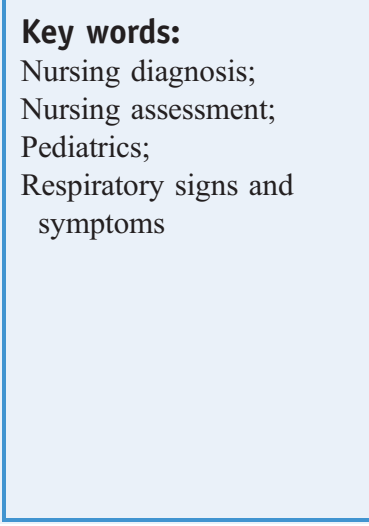

\section{Key words:}

Nursing diagnosis;

Nursing assessment;

Pediatrics;

Respiratory signs and symptoms

\begin{abstract}
The aim of this study was to identify the defining characteristics that allow clinical differentiation of the nursing diagnoses, ineffective breathing pattern (IBP), ineffective airway clearance (IAC), and impaired gas exchange (IGE). A secondary analysis with a cohort design was developed from 1128 records obtained during the hospital stay of 136 children with acute respiratory infection. Groups of defining characteristics with greater differentiation capacity were identified by multiple correspondence analyses. The results showed that the defining characteristics that better differentiate the studied diagnoses are agitation, irritability and diaphoresis for IGE; dyspnea, use of accessory muscles to breathe, orthopnea, and abnormal breathing pattern for IBP and excessive sputum, absence of cough, difficulty verbalizing, nasal flaring, and adventitious breath sounds for IAC. Twelve defining characteristics that can assist clinicians to differentiate the three main respiratory nursing diagnoses among children with acute respiratory infection were identified in this study.
\end{abstract}

(C) 2016 Elsevier Inc. All rights reserved.
STUDIES DEALING WITH nursing diagnoses and their components can produce data that help nurses to examine the way in which the evidence fits a certain diagnosis. This type of study facilitates the use of nursing diagnoses to provide nurses with a diagnostic language. Strategies aimed at such an approach are recommended and encouraged in view of their contribution to improving the skills used by nurses in the diagnostic reasoning process (Lunney, 2008).

\footnotetext{
* Corresponding author: Marcos Venícios de Oliveira Lopes, RN, PhD Statistician.

E-mail address:marcos@ufc.br.
}

Thus, studies aimed to differentiate between inter-related nursing diagnoses are useful to reduce the uncertainties that may arise during the diagnostic inference process. The implication of such diagnostic processes can be difficult if the diagnoses have similar characteristics or require the incorporation of information obtained from others. This problem can be worsened if the nurse does not know the relevance of each defining characteristic for each diagnosis. Moreover, even specific characteristics for a diagnosis may not necessarily be effective in multiple scenarios of clinical practice to allow for a differential diagnosis in a clinically uncertainty situation.

The nursing diagnoses, ineffective breathing pattern (IBP - Code 00032), ineffective airway clearance (IAC - 
Code 00031), and impaired gas exchange (IGE - Code 00030) represent human responses characterized by respiratory impairment that have strong clinical relationships to each other. These diagnoses share the defining characteristics of dyspnea, orthopnea, restlessness, cyanosis and nasal flaring, and they have other characteristics that are similar or incorporate parts of the information from other characteristics. For example, abnormal breathing pattern is a defining characteristic of IGE and is related indirectly to the characteristics of tachypnea, orthopnea, and dyspnea that comprise the IBP diagnosis (Herdman \& Kamitsuru, 2014).

Despite the fact that the diagnoses of IBP, IAC, and IGE exhibit similar defining characteristics, they were developed from the concepts of ventilation, permeability and gas exchange. Thus, it is conjectured that the physiological relationship between these concepts generates shared defining characteristics and directly influences the establishment of nursing interventions and the achievement of positive outcomes of care.

Therefore, the establishment of defining characteristics with good discriminating ability minimizes the discrepancies in clinical evaluations and assists in the identification of the nursing diagnosis that represents the true clinical condition. Generally, nurses identify a set of defining characteristics and verify the relationship between the most plausible diagnoses of a particular situation with the defining characteristics presented by the patient (Lopes, Silva, \& Araujo, 2012). Identification of each new characteristic can confirm a suspected diagnosis, eliminate another, or even redirect the nurse's attention to a human response not yet considered. At this point, the degree of knowledge of the relevance of each characteristic in regard to the diagnostic hypotheses assists with a more precise identification of the most credible nursing diagnoses for a specific clinical condition.

The prevalence of respiratory nursing diagnoses in individuals with different types of disease is relevant to nursing care because of the relationship between the respiratory system and other vital processes. Additionally, some populations are particularly sensitive to respiratory changes, such as children with acute respiratory infection (ARI) (Fleming, Pannell, \& Cross, 2005). Although the ARIs include a wide range of diseases with different clinical spectra, previous studies have reported the joint or isolated occurrence of IBP, IAC, and IGE. However, few studies have aimed at establishing differentiating clinical indicators for these nursing diagnoses.

Pascoal et al. (2012) assessed children with ARI and found that at least $42 \%$ of the samples had a joint occurrence of IBP, IAC, and IGE on the first day of evaluation. However, the isolated prevalence of each diagnosis reached $91.9 \%$ for IAC, $64 \%$ for IBP, and $42.6 \%$ for IGE. Another study that also evaluated children with ARI found a relatively high prevalence of IBP (59.6\%), IAC, (37.7\%), and IGE (27.2\%), but it did not describe the percentage of children who presented concomitantly with the three diagnoses (Andrade, Chaves, Silva, Beltrão, \& Lopes, 2012).
ARI corresponded to the most common group of respiratory diseases of childhood (Silva, Paiva, Silva, \& Nascimento, 2012) and caused changes that negatively impacted the respiratory system. Several factors contribute to the increased susceptibility of a child to the development of respiratory problems, including anatomical peculiarities and physiological and immunological characteristics (Matsuno, 2012). Therefore, these respiratory changes contribute to the development of characteristic signs and symptoms of respiratory nursing diagnoses in several clinical spectra.

The clinical amplitude ranges from a mild respiratory impairment to global impairment of respiratory function and induces the need for specific care plans for each condition that, when defined improperly, can compromise the care outcomes. Thus, the aim of this study was to identify which defining characteristics which all allow clinical differentiation of IBP, IAC, and IGE in children with ARI.

\section{Methods \\ Design and Sample}

This is a secondary analysis using a cohort design, developed to establish clinical parameters of differentiation of the IBP, IAC, and IGE nursing diagnoses based on the strength of association among their defining characteristics. The data consisted of 1128 records obtained from a cohort of 136 children with ARI followed for a period of hospitalization ranging between six and ten days in two pediatric hospitals in northeastern Brazil. The ethics committee of the institutions evaluated and approved the study, and parents or guardians were informed about the objectives of the study and consented to the data collection and signed an informed consent form.

The original cohort consisted of children aged between zero and five years (mean: 20.35 months, $\mathrm{SD}=3.11$ ) and admitted to the hospital in less than 48 hours; most of the subjects were male $(58.1 \%)$ and diagnosed with pneumonia (85.3\%), although other less severe ARI were identified, such as bronchiolitis, sinusitis, pharyngitis and tonsillitis. Children with chronic diseases that altered the specific clinical status of the ARI (e. g., congenital heart disease, cerebral palsy) were excluded from the original cohort.

The sample size was based on the recommendation of Menexes and Papadimitriou (2004) for studies with multiple categorical variables, which suggest that the minimum sample size should be calculated from the number of variables in pairs (q) by the formula: q. $(q-1) / 2$. The present study included 30 defining characteristics that make up the three nursing diagnoses. Thus, a minimum sample of 435 records $(30 \times 29 / 2)$ would be required to perform the analysis. We decided to work with all available records of the cohort, totaling 1128 observations.

\section{Data Collection}

The data of the original cohort were collected from an instrument based on the defining characteristics of IBP, IAC, and IGE, as described in NANDA International terminology 
(NANDA-I) (Herdman \& Kamitsuru, 2014) and in the relevant literature on pulmonary evaluation (Jarvis, 2011; Potter \& Perry, 2012). Operational definitions were created for each defining characteristic studied, and the data were collected by members of a research group on nursing diagnoses who had previously completed to an eight-hour training for review and standardization of the diagnostic procedures used in respiratory evaluation.

This analysis included 27 defining characteristics, six for IBP (tachypnea, use of accessory muscles to breath, increase in anterior-posterior chest diameter, altered chest excursion, pursed-lip breathing, and bradypnea), seven for IAC (ineffective cough, adventitious breath sounds, diminished breath sounds, excessive sputum, absence of cough, difficulty verbalizing, and wide-eyed look), eight for IGE (hypoxemia, tachycardia, abnormal skin color, irritability, diaphoresis, somnolence, headache upon awakening, and confusion), and six common to two or more diagnoses (abnormal breathing pattern, dyspnea, orthopnea, restlessness, nasal flaring, and cyanosis), as described in the NANDA-I terminology. The defining characteristics related to spirometric tests and blood gas tests were not evaluated because there were no data available for laboratory exams and/or there were medical contraindications for these exams.

\section{Data analysis}

Statistical analysis was performed with the support of the Statistical Package for the Social Sciences (SPSS) version 21.0, and the $\mathrm{R}$ software version 3.1. The differentiation capacity of the defining characteristics for the three nursing diagnoses studied was defined using multiple correspondence analysis. This type of analysis allows us to identify the dimensions of the relationships between categorical variables (Greennacre, 2007). In this study, defining characteristics were analyzed for their relationships and consequent clustering in dimensions. The objective was to identify groups of defining characteristics that allow discrimination between the three nursing diagnoses in question. In this case, the dimensions are supposed to represent these three nursing diagnoses.

Eigenvalues and inertia of the variance explained by each dimension were analyzed to evaluate the number of dimensions (nursing diagnoses) which best fit the data set (defining characteristics). Eigenvalues represent absolute values of the variance of each dimension, while inertia refers to the variance in relative terms, i.e., the inertia is obtained by the eigenvalue divided by the number of variables. The inertia varies from zero to one, and higher values represent a higher amount of variance explained by a specific dimension.

The determination of the quantity of dimensions that best fit the defining characteristics was initially based on the explained variance calculated for the maximum possible number of dimensions (equivalent to the total number of defining characteristics), and the discrimination measures for each defining characteristic. These discrimination measurements represent an optimized quantification of the association between each variable (defining characteristic) and each dimension (nursing diagnosis).

Following the initial solution with all possible dimensions, it was found that the solution in three dimensions presented the best results. Thus, a new multiple correspondence analysis based on only three dimensions was performed, new discrimination measures were calculated, and a two-dimensional graph was constructed. Defining characteristics that had values below 0.01 for discrimination measures in all dimensions, or those with similar values to two or more dimensions, were excluded from the final model. The division of the defining characteristics by dimension was defined based on the values of the discrimination measures and the clinical relationship of them with others in one dimension to adequately represent the main concept of the nursing diagnosis (ventilation, permeability or gas exchange). In addition, a twodimensional graph was constructed based on discrimination measures to assist in the visualization of the defining characteristics with higher discriminative capacity among the dimensions (nursing diagnoses).

\section{Results}

All 27 defining characteristics evaluated were identified in the sample, and the observed frequencies ranged from $88.7 \%$ (ineffective cough) to $0.1 \%$ (confusion). Two of the four defining characteristics that presented more frequently than $50 \%$ were components of the IAC diagnosis (ineffective cough and adventitious breath sounds), one was an IBP component (tachypnea) and one was integrated within these two diagnoses (abnormal breathing pattern). Characteristics associated with IGE showed frequencies lower than $30 \%$ in the sample, the most common being hypoxemia (28.6\%; 95\% CI: 26.0-31.4\%) (Table 1).

Table 2 summarizes the findings for the final model of multiple correspondence analysis. Dimension 1 showed the highest values for the measures of discrimination and included four defining characteristics (abnormal breathing pattern, use of accessory muscles to breath, dyspnea, and orthopnea), which comprised the IBP diagnosis, although two of these characteristics were common to other diagnoses. Moreover, these common characteristics are directly related to respiratory ventilation, which is the concept of concern within the IBP diagnosis.

Dimension 2 was composed of the largest number of defining characteristics (adventitious breath sounds, difficulty verbalizing, excessive sputum, nasal flaring, and absence of cough). In this dimension, the characteristics "adventitious breath sounds" and "absence of cough" had higher discriminatory measures for other dimensions. However, these characteristics were retained in dimension 2 for their clinical relationship with the concept of respiratory permeability and their specificity with a diagnosis of IAC.

Dimension 3 was composed of three defining characteristics (restlessness, irritability, and diaphoresis), and only diaphoresis showed low values for this dimension and was 
Table 1 Frequency of defining characteristics found in children's records $(n=1128)$.

\begin{tabular}{|c|c|c|c|}
\hline Defining characteristics & $\mathrm{n}$ & $\%$ & $95 \% \mathrm{CI}$ \\
\hline Ineffective cough ${ }^{\mathrm{a}}$ & 866 & 88.7 & $86.5-90.6$ \\
\hline Abnormal breathing pattern & 775 & 68.7 & $65.9-71.4$ \\
\hline Adventitious breath sounds & 734 & 65.1 & $62.2-67.8$ \\
\hline Tachypnea & 629 & 55.8 & $52.8-58.7$ \\
\hline Dyspnea & 516 & 45.7 & $42.8-48.7$ \\
\hline Use of accessory muscles to breath & 433 & 38.4 & $35.5-41.3$ \\
\hline Orthopnea & 385 & 34.1 & $31.4-37.0$ \\
\hline Diminished breath sounds & 384 & 34.0 & $31.3-36.9$ \\
\hline Hypoxemia & 323 & 28.6 & $26.0-31.4$ \\
\hline $\begin{array}{l}\text { Increase in anterior-posterior } \\
\text { chest diameter }{ }^{\text {b }}\end{array}$ & 306 & 27.7 & $25.1-30.5$ \\
\hline Excessive sputum $^{\mathrm{c}}$ & 282 & 26.9 & $24.3-29.7$ \\
\hline Tachycardia & 249 & 22.1 & $19.7-24.6$ \\
\hline Abnormal skin color & 169 & 15.0 & $13.0-17.2$ \\
\hline Restlessness & 158 & 14.0 & $12.0-16.2$ \\
\hline Absence of cough & 152 & 13.5 & $11.6-15.6$ \\
\hline Irritability & 117 & 10.4 & $8.7-12.3$ \\
\hline Difficulty verbalizing & 105 & 9.3 & $7.7-11.2$ \\
\hline Diaphoresis & 88 & 7.8 & $6.3-9.6$ \\
\hline Somnolence & 72 & 6.4 & $5.1-8.0$ \\
\hline Headache upon awakening & 53 & 4.7 & $3.6-6.1$ \\
\hline Nasal flaring & 36 & 3.2 & $2.3-4.4$ \\
\hline Altered chest excursion & 13 & 1.2 & $0.6-2.0$ \\
\hline Cyanosis & 7 & 0.6 & $0.2-1.3$ \\
\hline Pursed-lip breathing & 4 & 0.4 & $0.1-0.9$ \\
\hline Wide-eyed look & 3 & 0.3 & $0.1-0.8$ \\
\hline Bradypnea & 3 & 0.3 & $0.1-0.8$ \\
\hline Confusion & 1 & 0.1 & $0.0-0.5$ \\
\hline
\end{tabular}

maintained with the other two for presenting a more plausible clinical relationship with the other characteristics and the concept of gas exchange. Figure 1 provides a clearer view of the groups of defining characteristics for each of the dimensions shown in Table 2.

\section{Discussion}

Among the various nursing diagnoses of NANDA-I, studies highlight that diagnoses of IBP, IAC, and IGE have been frequently reported in the literature in different situations and age groups (Silva et al., 2011). The occurrence of respiratory nursing diagnoses in children with ARI can be explained because the infectious process, in addition to causing direct injury to the respiratory epithelium resulting in airway obstruction due to edema, abnormal secretions and cellular debris, also elevates airway resistance, causing a significant increase in respiratory work (Kleigman, Stanton, St Geme, Schor, \& Berhman, 2011).

Characteristics that have demonstrated the best discriminative capacity for the three diagnoses in this study were identified by frequencies ranging from $68.7 \%$ to $3.2 \%$. Some
Table 2 Multiple correspondence analyses for solution with three dimensions, including variables that presented the best discriminative capacity.

\begin{tabular}{|c|c|c|c|c|}
\hline \multirow{2}{*}{\multicolumn{2}{|c|}{ Defining characteristics }} & \multicolumn{3}{|c|}{ Dimension } \\
\hline & & 1 & 2 & 3 \\
\hline \multicolumn{2}{|c|}{ Abnormal breathing pattern } & 0.708 & 0.149 & 0.002 \\
\hline \multicolumn{2}{|c|}{ Use of accessory muscles to breath } & 0.527 & 0.080 & 0.076 \\
\hline \multicolumn{2}{|c|}{ Dyspnea } & 0.513 & 0.128 & 0.154 \\
\hline \multicolumn{2}{|l|}{ Orthopnea } & 0.450 & 0.149 & 0.100 \\
\hline \multicolumn{2}{|c|}{ Nasal flaring } & 0.102 & 0.053 & 0.031 \\
\hline \multicolumn{2}{|c|}{ Adventitious breath sounds } & 0.143 & 0.073 & 0.032 \\
\hline \multicolumn{2}{|c|}{ Difficulty verbalizing } & 0.024 & 0.073 & 0.007 \\
\hline \multicolumn{2}{|c|}{ Excessive sputum } & 0.046 & 0.051 & 0.010 \\
\hline \multicolumn{2}{|c|}{ Absence of cough } & 0.020 & 0.043 & 0.062 \\
\hline \multicolumn{2}{|c|}{ Irritability } & 0.162 & 0.235 & 0.572 \\
\hline \multicolumn{2}{|l|}{ Restlessness } & 0.201 & 0.241 & 0.511 \\
\hline \multicolumn{2}{|l|}{ Diaphoresis } & 0.096 & 0.115 & 0.078 \\
\hline \multirow[t]{2}{*}{ Dimension } & \multirow{2}{*}{$\begin{array}{l}\text { Cronbach's } \\
\text { alpha }\end{array}$} & \multicolumn{3}{|c|}{ Explained variance } \\
\hline & & \multicolumn{2}{|c|}{ Eigenvalues } & Inertia \\
\hline 1 & 0.846 & 5.498 & & 0.183 \\
\hline 2 & 0.631 & 2.561 & & 0.085 \\
\hline 3 & 0.515 & 1.991 & & 0.066 \\
\hline
\end{tabular}

characteristics with high frequencies, such as ineffective cough, tachypnea and diminished breath sounds, did not demonstrate good discriminatory power, while characteristics with frequencies below $10 \%$, such as diaphoresis, nasal flaring, and difficult verbalizing, formed subgroups representing different dimensions.

This study demonstrates that clinicians cannot restrict their clinical reasoning process to the observed frequency of defining characteristics. The quality of these characteristics and their more sensitive or specific relationship with the nursing diagnosis has been advocated as a guideline for clinical validation studies (Lopes et al., 2012). Thus, the ability of the defining characteristics to enable the differentiation of respiratory nursing diagnoses can be associated with their sensitivity and/or specificity for a specific diagnosis.

In a recent systematic review of clinical indicators of IBP, the five defining characteristics identified in the present study with best discriminative capacity showed statistically significant summary measures for the identification of IBP in a meta-analysis of six studies that addressed different pediatric populations (Sousa, Lopes, Silva, \& Keenan, 2015). Abnormal breathing pattern is a characteristic recently added to IBP in the NANDA-I terminology; this diagnosis includes changes in the breathing frequency, rate and respiratory depth. Although its scope might hinder comparisons, previous studies show that clinical signs related to an abnormal breathing pattern are often found in children diagnosed with IBP and among children with asthma and 


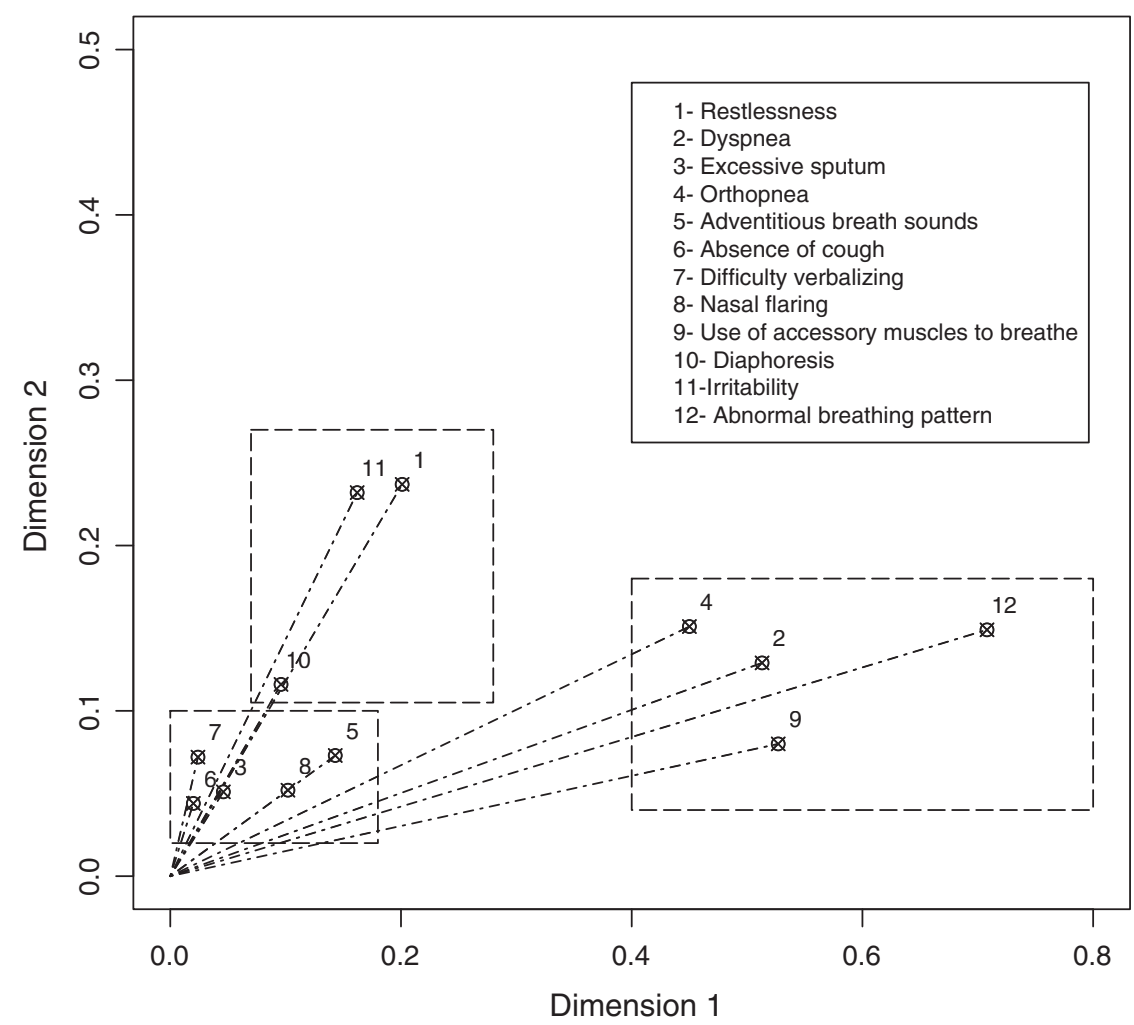

Figure 1 Groups of defining characteristics with the best discriminative capacity.

acute respiratory infections (Andrade et al., 2012; Cavalcante, Mendes, Lopes, \& Lima, 2010).

Dyspnea is a defining feature that has been associated with early stages of IBP among children with different cardiac and respiratory conditions. Previous studies have shown that dyspnea has high sensitivity in correctly identifying children with isolated ventilatory changes or in conjunction with other nursing diagnoses (Andrade et al., 2012; Beltrão et al., 2015; Cavalcante et al., 2010; Silveira, Lima, \& Lopes, 2008). On the other hand, the defining characteristics "use of accessory muscles to breathe" and "orthopnea" have been associated with IBP as confirmatory diagnostic characteristics because they have low sensitivity and high specificity values between children with asthma and acute respiratory infections (Andrade et al., 2012; Cavalcante et al., 2010).

Regarding the IAC, the characteristics of excessive sputum, absence of cough, difficulty verbalizing, and adventitious breath sounds are exclusive components of this diagnosis. Although this fact may partially explain the trend of these indicators to group together in one dimension and delimit its ability to discriminate this diagnosis from others, previous studies have shown that the characteristics of difficulty verbalizing, excessive sputum, and absence of cough exhibit high specificity for IAC, while the presence of adventitious breath sounds demonstrates high sensitivity for this nursing diagnosis among children with respiratory problems (Andrade et al., 2012; Mendes, Cavalcante, Lopes, \& Lima, 2012; Silveira et al., 2008). In addition, a recent systematic review of the diagnostic accuracy measures of the defining characteristics of IAC corroborated the findings of this study, showing that adventitious breath sounds and difficulty verbalizing presented statistical significance in the summary measures obtained from a meta-analysis that included five studies developed with children (Sousa, Lopes, \& Silva, 2015).

It is noteworthy that, although the characteristic "nasal flaring" is classified as an IBP and IGE component, the results of this study show that this characteristic has a good ability to discriminate for IAC. The presence of this defining characteristic in children with ARI can be explained by increased production of respiratory secretions and commitment in the defense mechanisms of the airways, which can lead to retention of secretions, excessive mucus secretion in the bronchi and exudate in the alveoli (Tarantino, 2013). Thus, nasal flaring can be a consequence of a more intense effort that is developed by the child to remove secretions and shows that this clinical indicator may be associated with a more severe spectrum of IAC.

For the IGE diagnosis, it is conjectured that the emphasis on the defining characteristics "restlessness" and "irritability" is linked to the fact that they consist of symptoms associated with respiratory disorders with cerebral oxygen deprivation. The physiological mechanism involved in these symptoms is based on the reduction of the perfusion of the central nervous system due to hypoxia (Kleigman et al., 2011). However, it is important to note that because this research was conducted 
with data from children under the age of five years, the results obtained should be used with caution given that other situations (hospital environment, hunger, sleep, and contact with strangers) can also lead to the manifestation of restlessness and irritability. Another important relationship with IGE is the presence of hypercapnia, which exerts a stimulus on the adrenal medulla, increasing the secretion of catecholamines, epinephrine and norepinephrine, accelerating cell metabolism and the production of heat, and triggering diaphoresis (Guyton \& Hall, 2011).

Previous studies have demonstrated a high specificity of these three defining characteristics for the identification of IGE (Andrade et al., 2012; Sousa et al., 2015). In addition, a systematic review included three studies about the accuracy of defining characteristics for IGE and found statistically significant summary measures for restlessness and irritability, confirming the importance of these characteristics to diagnose changes in gas exchange processes (Sousa, Lopes, \& Silva, 2014).

Clinical alterations caused by acute respiratory infection directly influence the establishment of the nursing diagnoses IBP, IAC, and IGE. Thus, the findings of this study should be analyzed in light of some limitations. The lack of control of data collection and targeting of variables to the research question affect a secondary analysis of data. Thus, the absence of data from spirometry tests prevented the inclusion of some defining characteristics and may have affected the results in the final model of multiple correspondence analysis. Another important point is that the age of children in the original cohort made it difficult to obtain data on some defining characteristics, because it was necessary to obtain information directly from parents/guardians. This situation is particularly sensitive to recall bias and leads to low accuracy of the presented estimates. Finally, it has been highlighted that the lack of studies in the literature with methodological approaches similar to this research made it difficult to compare results, indicating the need for further research on this topic.

The results of this study can contribute to a process of accurate diagnostic inference to provide information allowing the discrimination between three major nursing diagnoses for children with acute respiratory infection. In addition, educators and nursing students will have a better understanding of the information needed to assess the presence of nursing diagnoses and to directly test hypotheses related to diagnostic differentiation among IBP, IAC, and IGE.

\section{Acknowledgment}

Financial support: Fundação de Amparo à Pesquisa e ao Desenvolvimento Científico e Tecnológico do Maranhão (FAPEMA).

\section{References}

Andrade, L. Z. C., Chaves, D. B. R., Silva, V. M., Beltrão, B. A., \& Lopes, M. V. O. (2012). Respiratory nursing diagnoses for children with acute respiratory infection. Acta Paulista de Enfermagem, 25, 713-720, http://dx.doi.org/10.1590/S0103-21002012000500011.
Beltrão, B. A., Herdman, T. H., Pascoal, L. M., Chaves, D. B. R., Silva, V. M., \& Lopes, M. V. O. (2015). Ineffective breathing pattern in children and adolescents with congenital heart disease: Accuracy of defining characteristics. Journal of Clinical Nursing, 24, 2505-2513, http://dx. doi.org/10.1111/jocn.12838.

Cavalcante, J. C. B., Mendes, L. C., Lopes, M. V. O., \& Lima, L. H. O. (2010). Clinical indicators of ineffective breathing pattern in children with asthma. Revista da Rede de Enfermagem do Nordeste, 11, 66-75.

Fleming, D. M., Pannell, R. S., \& Cross, K. W. (2005). Mortality in children from influenza and respiratory syncytial virus. Journal of Epidemiology and Community Health, 59, 586-590.

Greennacre, M. (2007). Correspondence analysis in practice (2nd ed.). London: Chapman \& Hall.

Guyton, A. C., \& Hall, J. E. (2011). Guyton \& Hall: Textbook of medical physiology (12th ed.). Philadelphia: W.B. Saunders.

Herdman, T. H., \& Kamitsuru, S. (Eds.). (2014). NANDA International nursing diagnoses: Definitions and classification, 2015-2017. Oxford, UK: Wiley-Blackwell.

Jarvis, C. (2011). Physical examination and health assessment (6th ed.). St Louis, MO: Elsevier Saunders.

Kleigman, R. M., Stanton, B. F., St Geme, J. W., Schor, N. F., \& Berhman, R. E. (2011). Nelson textbook of pediatrics (19th ed.). St Louis, MO: Elsevier Saunders.

Lopes, M. V. O., Silva, V. M., \& Araujo, T. L. (2012). Methods for establishing the accuracy of clinical indicators in predicting nursing diagnoses. International Journal Nursing Knowledge, 23, 134-139, http://dx.doi.org/10.1111/j.2047-3095.2012.01213.x.

Lunney, M. (2008). Current knowledge related to intelligence and thinking with implications for the use and development of case studies. International Journal Nursing Terminology Classification, 19, 158-162, http://dx.doi.org/10.1111/j.1744-618X.2008.00104.x.

Matsuno, A. K. (2012). Respiratory failure in children. Medicina, 45, 168-184.

Mendes, L. C., Cavalcante, J. C. B., Lopes, M. V. O., \& Lima, L. H. O. (2012). Ineffective airway clearance in children with asthma: a descriptive study. Texto \& Contexto Enfermagem, 21, 371-378, http:// dx.doi.org/10.1590/S0104-07072012000200015.

Menexes, G., \& Papadimitriou, I. (2004). Relations of inertia in simple contingency, generalized contingency (Burt) and indicator matrices for two or more variables. Workbooks of Data Analysis, 4, 42-69.

Pascoal, L. M., Beltrão, B. A., Chaves, D. B. R., Lopes, M. V. O., Silva, V. M., Sousa, V. E. C., et al. (2012). Estudio longitudinal de los diagnósticos de enfermería respiratorios en niños con infección respiratoria aguda. Enfermería Clínica, 22, 255-260, http://dx.doi.org/ 10.1016/j.enfcli.2012.06.004.

Potter, P. A., \& Perry, A. G. (2012). Fundamentals of nursing (8th ed.). St Louis: MO:Elsevier.

Silva, V. M., Lopes, M. V. O., Araujo, T. L., Beltrão, B. A., Monteiro, F. P. M., Cavalcante, T. F., et al. (2011). Operational definitions of outcome indicators related to ineffective breathing patterns in children with congenital heart disease. Heart \& Lung, 40, 70-77, http://dx.doi.org/10. 1016/j.hrtlng.2010.12.002.

Silva, M. D. B., Paiva, M. B., Silva, L. R., \& Nascimento, M. A. L. (2012). Acute respiratory disease in the child: an integrative review. Revista Enfermagem UERJ, 20, 260-266.

Silveira, U. A., Lima, L. H. O., \& Lopes, M. V. O. (2008). Características definidoras dos diagnósticos de enfermagem desobstrução ineficaz das vias aéreas e padrão respiratório ineficaz em crianças asmáticas. Revista da Rede de Enfermagem do Nordeste, 9, 125-133.

Sousa, V. E. C., Lopes, M. V. O., \& Silva, V. M. (2014). Meta-analisi dell'accuratezza delle caratteristiche definenti di Compromissione degli scambi gassosi. Assistenza Infermieristica e Ricerca, 33, 29-35.

Sousa, V. E. C., Lopes, M. V. O., \& Silva, V. M. (2015). Systematic review and meta-analysis of the accuracy of clinical indicators for ineffective airway clearance. Journal of Advanced Nursing, 71, 498-513, http://dx. doi.org/10.1111/jan.12518.

Sousa, V. E. C., Lopes, M. V. O., Silva, V. M., \& Keenan, G. M. (2015). Defining the key clinical indicators for ineffective breathing pattern in 
paediatric patients: A meta-analysis of accuracy studies. Journal of Clinical Nursing, 24, 1773-1783, http://dx.doi.org/10.1111/jocn.12815.

Sousa, V. E. C., Pascoal, L. M., Matos, T. F. O., Nascimento, R. V., Chaves, D. B. R., Guedes, N. G., \& Lopes, M. V. O. (2015). Clinical indicators of impaired gas exchange in cardiac postoperative patients. International Journal of Nursing Knowledge, 26, 141-146, http://dx.doi.org/10.1111/2047-3095.12061.

Tarantino, A. B. (2013). Sistema respiratório. In PORTO, C.C. (Ed.), Semiologia Médica. Rio de Janeiro: Guanabara Koogan. 